\title{
"CONCEPCIONES DE CIENCIA E INVESTIGACIÓN QUE SUBYACE EN LOS PROFESORES DE CIENCIAS, ASPIRANTES AL PROGRAMA DE MAESTRÍA EN DOCENCIA DE LA QUÍMICA"
}

\author{
Manuel Erazo Parga. merazop@uni.pedagogica.edu.co \\ Programa de Maestría en Docencia de la Química. \\ Universidad Pedagógica Nacional
}

\begin{abstract}
SUMMARY: In this work is intended to investigate about the influence of the philosophical theories of empirical positivism and psychological behaviorism that still persist in the mind of the science teachers and that in some way can influence about their science conceptions, production of knowledge, about the teaching learning process, which in some way affects the student evaluation. About the problems mentioned above there are several papers in many publications in magazines and what is wanted is to show that it also affects the professors applicants to the graduate degree program in teaching of the chemistry. The abovementioned defines the necessity to offer a program with investigation lines to find solutions and new didactic alternatives so that the professor modifies his traditional methodologies.
\end{abstract}

GLOSARY: philosophical empiropositivismo, psychological behaviorismo, Scientific knowledge, teaching and learning.

RESUMEN: En este trabajo se pretende investigar sobre la influencia de las teorías filosóficas empírico positivistas y el conductismo psicológico que todavía persisten en la mente de los profesores de ciencias y que de alguna manera puede influenciar sus concepciones sobre la ciencia y los procesos de producción del conocimiento científico, así como también sobre el proceso de enseñanza aprendizaje y de las formas de evaluar a los estudiantes. Sobre estos problemas hay varias publicaciones en revistas y lo que se quiere es mostrar que también afecta a los profesores aspirantes al programa de postgrado en enseñanza de la química. Lo anterior define la necesidad de ofrecer un programa con líneas de investigación para encontrar soluciones y nuevas alternativas didácticas para que el profesor modifique sus metodologías tradicionales.

GLOSARIO: empiropositivismo filosófico, conductismo sicologico, conocimiento científico, enseñanza y aprendizaje

\section{INTRODUCCION}

Uno de los presupuestos teóricos relacionados con las concepciones de los profesores de Ciencias en ejercicio y para quienes está dirigido el programa de Maestría en Docencia de la Química, es que estos, en su práctica profesional, están de alguna manera influenciados por las corrientes filosóficas positivistas y psicológico conductistas.

Esta influencia afecta su pensamiento y toma de decisiones, en relación con la ciencia y los procesos de producción de conocimiento así como sus concepciones de enseñanza, aprendizaje y los fines que este proceso persigue en el sistema educativo.

En el presente artículo se reportan los resultados obtenidos de la aplicación de un instrumento de 15 afirmaciones relacionadas con la ciencia y sus procesos de producción de conocimiento, aplicado al comenzar cada una de las últimas 6 promociones que iniciaron el programa de Maestría en Docencia de la Química.

\section{DESARROLLO}




\section{Antecedentes}

Cabe señalar la importancia que tiene hoy en día la línea de investigación "El Pensamiento del Profesor", cuyo objeto de estudio está orientado hacia la caracterización de los diferentes componentes que influyen en el pensamiento de los profesores de ciencias, desde los cuales toma decisiones en su ejercicio profesional y que a su vez están relacionados con lo que él cree debe saber, saber hacer y saber ser como profesor miembro de una comunidad educativa y responsable de un proceso educativo de calidad.

Hacia mediados de la década de los 80 s, las investigaciones sobre el estudio de las conductas del profesor, su formación basada en competencias técnicas para la enseñanza, etc que formaban parte de los problemas a resolver por los investigadores en este campo desde la década de los 70 s, cambió hacia el estudio de su pensamiento y de su formación, observándose un progreso en las investigaciones sobre lo que los profesores piensan acerca de la ciencia, su enseñanza y aprendizaje.

En estos trabajos se destaca, en primer lugar, el hecho de que los profesores tienden a exaltar la supremacia del conocimiento científico sobre otros conocimientos (Lederman 1992) y en segundo lugar revelan que los profesores de ciencias presentan diversidad de concepciones sobre la ciencia entre los que se destacan tres tendencias epistemológicas, racionalismo, relativismo y empirismo donde esta última posición es la mayoritariamente observada, y las relativistas son las más escasas (Porlán 1989). De la misma manera, Mellado (1996) señala sobre la inexistencia entre los profesores de una concepción definida y coherente sobre el conocimiento científico, siendo lo más destacable la escasa formación epistemológica del profesor.

Según Furió y otros (1992) este conocimiento de los profesores constituye un "pensamiento docente de sentido común" adquirido generalmente por el ambiente de formación durante su proceso de formación inicial y que tiene gran influencia en la actuación futura de éstos.

En la actualidad, existe un consenso creciente en torno a la necesidad de investigar sobre la epistemología "espontánea" del profesorado, dado que la misma puede constituirse en un obstáculo capaz de bloquear los intentos de renovación de la enseñanza de las ciencias. Por tal razon, las investigaciones en torno a las concepciones de los profesores, en relación con la ciencia, sus procesos de producción y su enseñanza, son hoy en día una línea de investigación de la que son exponentes numerosos artículos y tesis doctorales Porlan 1989; Praia 1995; Paixao 1998; Fernnández 2000, (Fernández y otros 2002).

En investigaciones últimamente realizadas, en Colombia, en el campo de las concepciones que sobre algunos conceptos científicos poseen los profesores, (Goodwin A; Orlik Y, 2002) así como también las concepciones de ciencia y de su enseñanza por parte de dichos profesores, han permitido caracterizar que el desempeño de estos en gran parte continúa siendo influenciado por el positivismo filosófico y el conductismo psicológico donde se destaca la idea de la observación como base del conocimiento científico y a la experimentación como el único instrumento para comprobar las teorías ya existentes. (Erazo P. M 1999).

Estas concepciones que mantienen los profesores de química obedecen en parte a que los programas de formación inicial y permanente de profesores, muestran la existencia de un esquema de trabajo predominante de enseñanza y aprendizaje por transmisión-asimilación. Gracias a la investigación didáctica se han podido detectar dichos esquemas, por lo que se hace necesario identificar en qué medida estas tendencias se siguen presentando en 
profesores de química en ejercicio y especialmente en los aspirantes al programa MDQ (Maestría en Docencia de la Química de la Universidad Pedagógica Nacional).

Para superar estas situaciones es necesario facilitar un proceso de formación inicial del profesor que le permita avanzar desde el nivel en que este se encuentran hasta llegar a convertirlo en un profesional autónomo, reflexivo crítico e investigador (Martínez Aznar y otros 2001), en otras palabras hacer de los programas de formación inicial y continua espacios para posibilitar que el futuro profesor sea un facilitador del aprendizaje significativo de los alumnos y un investigador de los procesos de enseñanza aprendizaje que se dan en su aula.

En síntesis, un programa de postgrado y en especial los de Maestría, deberán estar orientados a la formación investigativa del profesor en el campo de la didáctica, para generar mecanismos metodológicos de investigación que hagan de la actividad investigativa la columna vertebral para la formación continua y permanente del profesor para que identifique nuevas y mejores alternativas de actuación en el aula y posibilite el rompimiento con sus esquemas tradicionales.

A este respecto, el programa de Maestría en Docencia de la Química propende por:

- Promover el desarrollo profesional de los profesores de química en ejercicio.

- Potenciar el desarrollo de destrezas metodológicas e investigativas que articulen una teoría y una práctica coherentes dentro de un contexto social determinado.

- Establecer un concepto de desarrollo y/o renovación profesional, basado en la reflexión sobre el propio trabajo que pueda ser requerido a lo largo de la vida profesional.

- Formar a los profesores para que puedan valorar y responder constructivamente a las dificultades que aparecen en la práctica, incluyendo iniciativas organizativas y políticas.

- Preparar al profesorado para formar parte de una comunidad de especialistas más profesional y efectiva.

\section{Referente Teórico}

A partir de la investigación didáctica actual se ha introducido un replanteamiento en las visiones curriculares, a la vez que se ha llegado al convencimiento de que, en los alumnos, las actitudes científicas se forman dependiendo de la imagen que el profesor tenga de la ciencia y de sus procesos de producción, determinantes igualmente en las metodologías de enseñanza por éste empleadas.

Igualmente, las modernas tendencias de la Filosofía de las ciencias han indicado y subrayado de un modo muy preciso las dificultades profundamente arraigadas que están asociadas a la idea de que la ciencia se basa en un seguro fundamento adquirido gracias a la observación y a la experimentación, y a la idea de que hay cierto tipo de procedimiento inferencial que nos permite derivar teorías científicas de una manera fiable.

Con frecuencia, muchos saberes y quehaceres cotidianos se tildan de científicos con el argumento ingenuo de que éstos, autodenominados "saberes científicos", sí lo son porque siguen el método empírico de la física, que para quienes apoyan ésta tesis, dichos saberes y quehaceres son el producto consistente en la recopilación de "hechos" mediante una "observación" y una "experimentación" cuidadosa a partir de lo cual se derivaron posteriormente leyes y teorías de éstos hechos mediante una serie de procedimientos lógicos. 
Hoy tenemos claro que no hay método alguno, que permita probar que las teorías científicas son verdaderas, ni siquiera, probablemente verdaderas.

Existen al respecto diversas corrientes de pensamiento en relación con la ciencia y los procesos de producción de conocimiento por ésta empleados, los cuales influyen determinantemente en las conductas de los científicos y especialmente en aquellas personas que como los profesores deben trabajar por el desarrollo científico, los intereses, las actitudes y las transformaciones intelectuales de las juventudes.

Para superar éstas dificultades, ha de darse entonces un enfrentamiento conceptual y metodológico entre las estructuras de las ciencias, la de los profesores y la de los estudiantes, con el fin de que unos y otros hagan las críticas conceptuales, la revisión de los fundamentos, logren el desarrollo intelectual requerido y produzcan las reconstrucciones, necesarias para la adopción significativa y crítica del paradigma científico, alrededor del cual se monta el trabajo didáctico en el aula.

\section{Los errores de la Enseñanza Tradicional.}

Una de las razones por las que la enseñanza expositiva tradicional no logra que los alumnos comprendan mínimamente la ciencia, radica en la concepción estática del conocimiento científico que está vigente aún en los libros de texto y por supuesto, en la mente del profesor. Al alumno se le trasmite información desvirtuada y/o acabada, se le enseña la verdad científica que él debe reproducir. Esta transmisión de saberes cerrados en sí mismos para su reproducción es contraria, no solo al propio origen histórico de esos conocimientos, sino también a los mecanismos de aprendizaje de que dispone el alumno.

Es conocido que el aprendizaje es un proceso activo que exige del alumno la puesta en acción de sus esquemas de conocimiento para asimilar la realidad. En otras palabras, se aprende comprendiendo, es decir asimilando activamente las nuevas concepciones a conocimientos ya disponibles.

Como es sabido, los alumnos tienen fuertes ideas previas con respecto a la mayor parte de los fenómenos científicos, ideas que se caracterizan por ser muy persistentes al cambio y por ser generalmente opuestas a las concepciones científicas que se les pretende enseñar. (Pozo 1987).

Por lo tanto la enseñanza tradicional al no activar esas ideas y al no trabajar a partir de ellas, las mantiene intactas, generando en el alumno, en el mejor de los casos dos tipos de conocimientos: uno "espontáneo", útil para solucionar problemas cotidianos y para entender el mundo que le rodea, y un segundo conocimiento "académico", asimilado superficialmente, ajeno a sus propias ideas y útil exclusivamente para resolver problemas escolares de un modo meramente mecánico y para aprobar los exámenes a que es sometido por el profesor.

Los profesores de ciencias que de alguna manera están influenciados por estas concepciones, no se esfuerzan por fomentar en el estudiante el desarrollo de habilidades en cuanto a la formulación de hipótesis ante un problema, combinar y controlar variables etc. entonces no le desarrolla las capacidades propias del pensamiento científico. En consecuencia, la sola exposición de la verdad científica a los alumnos, de una manera acrítica, no sólo no les proporciona una comprensión de los conceptos científicos ni les ayuda a elaborarlos por sí mismos, sino que además produce en ellos una visión deformada y estática de la construcción del conocimiento científico. 
Investigaciones recientes (Fernández I, y otros 2002) señalan que del mismo modo que los alumnos poseen preconcepciones, ideas y comportamientos intuitivos, que interfieren en el aprendizaje de los conocimientos científicos, cabe suponer también que los profesores poseen preconcepciones acerca de la ciencia, sus procesos de producción de conocimiento los cuales pueden entrar en conflicto con las concepciones de enseñanza y aprendizaje de las ciencias. Ignorar este conocimiento tiene los mismos efectos negativos que no considerar las preconcepciones de los alumnos a la hora de diseñar una determinada estrategia de aprendizaje.

Los resultados de las investigaciones sobre la influencia que los profesores ejercen en el proceso de implementación de los currículos muestran la necesidad de cuestionar y modificar las "teorías implícitas" del profesorado acerca de cómo aprenden los alumnos y de cual es la naturaleza del conocimiento científico, para que las propuestas fundamentadas de innovación de la enseñanza puedan ser llevadas correctamente a la practica.

Las visiones deformadas acerca de la ciencia que configuran el esquema conceptual de los profesores de ciencias y que han sido mayoritariamente estudiadas se señalan a continuación (Fernández I, y otros 2002):

\section{* Concepción empiroinductivista y ateórica de la ciencia.}

Destaca la importancia de la observación y de la experimentación "neutras" no contaminada por ideas apriorísticas, olvidando el papel de las hipótesis como hilo conductor focalizante del proceso investigativo y de los referentes teóricos como orientadores de todo el proceso. En un intento de renovación de estos posicionamientos, se incurre en aprendizaje por descubrimiento que es una visión ateórica centrada en el supuesto "método científico".

* Concepción rígida de la actividad científica.

Se caracteriza por una visión rígida y dogmática, algorítmica, exacta e infalible de la actividad científica. Aquí el método científico se presenta como un conjunto de etapas a seguir de manera mecánica, destacando el rigor del mismo y el carácter exacto de los resultados obtenidos.

* Concepción aproblemática y ahistórica de la ciencia.

Se plantea el desarrollo del conocimiento científico como un discurso elaborado sin mostrar los problemas que generaron su construcción y su proceso evolutivo así como sus dificultades y limitaciones explicativas; perdiendo con esto toda posibilidad de presentar el conocimiento como respuesta a un cuestionamiento, a un problema, lo cual dificulta captar la racionalidad del proceso científico.

* Concepción exclusivamente analítica.

Resalta la necesaria parcelación inicial de los estudios, su carácter acotado, simplificatorio pero que olvida los esfuerzos posteriores de unificación y de construcción de cuerpos coherentes de conocimiento cada vez mas amplios. El olvido de estos procesos de unificación como característica fundamental de la evolución de los conocimientos científicos constituye un autentico obstáculo para la educación científica.

* Concepción meramente acumulativa del desarrollo científico.

Es una interpretación simplista de la evolución de los conocimientos científicos a la que la enseñanza suele contribuir al presentar los conocimientos hoy aceptados sin mostrar como dichos conocimientos han sido alcanzados ni referirse a las frecuentes confrontaciones entre 
teorías rivales. Transmite una visión acumulativa de crecimiento lineal de los conocimientos científicos, ignorando las crisis y las remodelaciones profundas, fruto de procesos complejos.

* Concepción individualista y elitista de la ciencia.

Los conocimientos científicos aparecen como obra de genios aislados, ignorándose el papel del trabajo colectivo, de los intercambios entre equipos. Se acepta la creencia de que los resultados obtenidos por un solo científico o equipo pueden bastar para verificar o falsar una hipótesis o incluso una teoría.

* Visión descontextualizada, socialmente neutra de la actividad científica.

Esta visión ignora, o trata superficialmente las complejas relaciones CTS o CTSA (ciencia tecnología sociedad y ambiente). Se reduce a la enumeración de algunas aplicaciones de los conocimientos científicos, cayendo así en una exaltación simplista de la ciencia como factor absoluto de progreso.

Para el caso de la presente investigación, se atenderá a la primera concepción señalada sobre la base de que los supuestos teóricos indican que los aspirantes al programa de Maestría en Docencia de la Química, la mayoría de ellos profesores en ejercicio en la educación básica y algunos en instituciones de educación superior poseen concepciones acerca de la ciencia y sus procesos de producción de conocimiento influenciados por posicionamientos filosóficos del tipo:

\section{Ingenuo - empiropositivista}

- Enmarcada por aquellas posiciones de los profesores en las que se manifiesta una ignorancia en cuanto al papel de la investigación en la construcción de la teoría. Se maneja una visión absolutista de la verdad, es decir, con la noción de que el conocimiento es una recopilación creciente de hechos comprobados o "conjunto de verdades".

- El crecimiento del conocimiento científico esta dado a través de la acumulación de observaciones.

- El progreso científico se manifiesta mediante la acumulación sucesiva de las teorías verdaderas.

- Las teorías son verdades sacadas de la naturaleza por observación objetiva de regularidades.

- El conocimiento es verdadero, duradero y universal, en correspondencia como el mundo funciona.

- La producción del conocimiento científico se debe al seguimiento de una serie de pasos secuenciales que van de la observación a la teoría.

- El método inductivo permite comparar teorías rivales, pues la naturaleza real tiene la última palabra.

- Lo que se le puede aplicar el método inductivo es científico.

\section{Inductivista - experimental}

- Cuando los profesores asocian la Ciencia con el estudio directo de la realidad mediante un método "objetivo": el método Científico, el cual es asumido como un conjunto de procesos y de pautas ordenadas de conducta que garantiza la objetividad y la eficacia del mismo: observación - hipótesis - teorías $(\mathrm{O}-\mathrm{H}-\mathrm{T})$, en consonancia con una visión absolutista de la verdad y del conocimiento.

\section{Hipótesis}


Con respecto a las concepciones que el profesor ha construido sobre la Ciencia y los procesos de producción de conocimiento científico (la Investigación), es posible encontrar que los aspirantes al programa posean un predominio de concepciones que oscilan entre una Concepción ingenua e intuitiva y una Concepción inductivista-experimental.

\section{Variables e indicadores}

De acuerdo con la naturaleza de la investigación la Variable Dependiente es "El pensamiento de los aspirantes al programa de Maestría en Docencia de la Química" desde el punto de vista de la Concepción de Ciencia e Investigación, que guían su ejercicio profesional, como variables Independientes.

Los indicadores corresponden al grado de aceptación o no por cada uno de los 15 ítems propuestos, desde una perspectiva empiro-positivista de la ciencia y sus procesos de producción.

\section{Metodología.}

La investigación sobre las concepciones epistemológicas de los profesores son hasta hoy las mas estudiadas y su estudio se puede abordar de diferentes maneras, que van desde el uso de diferentes tipos de cuestionarios, análisis de textos escolares, observación de clases y entrevistas con los profesores hasta seguimientos y acompañamientos mas permanentes y detallados que combinan varios instrumentos y procedimientos para la recolección de información. Este trabajo se centra en el análisis de un instrumento el cual posee una serie de afirmaciones en relación con la ciencia y sus procesos de producción de conocimiento, para que el profesor establezca el grado de acuerdo o desacuerdo en relación con la proposición que se le presenta

Población y Muestra: El universo de la población afectada por la investigación siguen siendo los Profesores de química adscritos al sistema educativo Colombiano y que ejercen la docencia al nivel de la educación básica secundaria y en instituciones de educación superior. La muestra corresponde a un total de 54 profesores en ejercicio y otros profesionales de las últimas seis promociones que han solicitado ingreso al programa de Maestría en Docencia de la Química

\section{Instrumento para recolectar Información.}

Está configurado por 15 ítems extraídos de 5 instrumentos aplicados en una investigación anterior similar y relacionada con las influencias del positivismo filosófico y conductismo psicológico en profesores de ciencias. En su versión inicial, los instrumentos fueron probados en grupo piloto y juicio de expertos, realizando las correspondientes pruebas de confiabilidad y validez.

Las afirmaciones en relación con la ciencia y sus procesos de producción de conocimiento están expuestos desde una perspectiva amigablemente empiropositivista y se pretende identificar el grado de acuerdo o desacuerdo que sobre estas afirmaciones tienen los profesores y demás profesionales de la química que solicitan ingreso al programa.

Para ello se tiene la siguiente escala de evaluación de cada ítem, que le plantea lo siguiente: Para cada afirmación que UD. encuentra en el documento adjunto, marque con una $\mathbf{X}$ en el lugar de su preferencia según la siguiente escala:

\begin{tabular}{|c|c|c|c|c|c|c|c|c|c|}
\hline 1 & $\begin{array}{c}\text { Totalmente } \\
\text { en }\end{array}$ & $\mathbf{2}$ & $\begin{array}{c}\text { En } \\
\text { Desacuerd }\end{array}$ & $\mathbf{3}$ & $\begin{array}{c}\text { Algo de } \\
\text { Acuerdo }\end{array}$ & $\mathbf{4}$ & De Acuerdo & $\mathbf{5}$ & $\begin{array}{c}\text { Totalmente } \\
\text { de Acuerdo }\end{array}$ \\
\hline
\end{tabular}


UNIVERSIDAD PEDAGOGICA NACIONAL

Desacuerdo $\mathbf{0}$

\begin{tabular}{|l|l|l|l|} 
& & & \\
\hline
\end{tabular}




\section{Resultados y análisis de resultados}

A continuación se presenta el instrumento aplicado y el grado de aceptación para cada ítem, con las correspondientes frecuencias y porcentajes.

Frecuencias de aceptación por ítem.

\begin{tabular}{|c|c|c|c|c|c|c|}
\hline \multicolumn{7}{|c|}{ Tabla No 1} \\
\hline $\mathbf{N}^{\circ}$ & Tamaño de la muestra: 54 aspirantes & TD & $\mathbf{D}$ & $\begin{array}{l}\text { D- } \\
\text { A }\end{array}$ & A & TA \\
\hline 1 & $\begin{array}{l}\text { La observación es el primer paso del método científico y está } \\
\text { relacionada con la forma como percibimos, con los órganos de } \\
\text { los sentidos, el mundo que nos rodea, esto nos permite admitir } \\
\text { la seguridad en nuestras apreciaciones y la confiabilidad en } \\
\text { los resultados. }\end{array}$ & 3 & 8 & 16 & 15 & 12 \\
\hline 2 & $\begin{array}{l}\text { La observación proporciona el acceso más confiable a un } \\
\text { conocimiento y asegura una concepción adecuada de la } \\
\text { realidad, de ésta manera se construyen las teorías actuales. }\end{array}$ & 13 & 22 & 9 & 5 & 5 \\
\hline 3 & $\begin{array}{l}\text { La actividad investigativa gira alrededor de un hecho o } \\
\text { fenómeno que ocurre en la realidad y que amerita una explica- } \\
\text { ción. }\end{array}$ & 2 & 2 & 2 & 27 & 21 \\
\hline 4 & $\begin{array}{l}\text { Las investigaciones que realizan los alumnos se deben centrar } \\
\text { fundamentalmente en las bibliotecas con el fin de aprender a } \\
\text { acceder a las fuentes de información. }\end{array}$ & 21 & 21 & 9 & 2 & 1 \\
\hline 5 & $\begin{array}{l}\text { Existe un método específico para la investigación, el cual nos } \\
\text { proporciona las pautas a seguir en relación con el objeto a } \\
\text { investigar y de acuerdo con su naturaleza, el investigador } \\
\text { realiza experimentos con el fin de encontrar la respuesta más } \\
\text { conveniente. }\end{array}$ & 6 & 14 & 14 & 14 & 6 \\
\hline 6 & $\begin{array}{l}\text { Los conceptos y las teorías son producidos por actos creativos } \\
\text { que surgen directamente de la observación y experimentación } \\
\text { rigurosa de la realidad. }\end{array}$ & 7 & 4 & 15 & 13 & 15 \\
\hline 7 & $\begin{array}{l}\text { Las explicaciones científicas pueden ser aceptadas como } \\
\text { verdaderas solamente si ellas pueden ser comprobadas por } \\
\text { observación y/o la experimentación. }\end{array}$ & 2 & 5 & 14 & 18 & 15 \\
\hline 8 & $\begin{array}{l}\text { Las teorías son el producto de la experimentación y el } \\
\text { experimento es necesario para darle sentido a las observacio- } \\
\text { nes de eventos naturales. }\end{array}$ & 2 & 3 & 15 & 21 & 13 \\
\hline 9 & $\begin{array}{l}\text { La teoría científica proporciona una descripción verdadera del } \\
\text { mundo. Se supone además que todas las explicaciones } \\
\text { científicas tienen que ver con fenómenos de existencia real. }\end{array}$ & 5 & 12 & 13 & 17 & 7 \\
\hline 10 & $\begin{array}{l}\text { Un principio que debe reconocer el profesor de ciencias es } \\
\text { que la observación y la experimentación son los procesos más } \\
\text { confiables y seguros a partir del cual se elaboran las teorías. }\end{array}$ & 4 & 6 & 9 & 22 & 13 \\
\hline
\end{tabular}

Digitalizado por RED ACADEMICA 


\begin{tabular}{|c|c|c|c|c|c|c|}
\hline 11 & $\begin{array}{l}\text { El observador científico no debe actuar bajo la influencia de } \\
\text { teorías previas sobre el problema investigado para no } \\
\text { parcializar sus conclusiones. }\end{array}$ & 13 & 17 & 14 & 7 & 3 \\
\hline 12 & $\begin{array}{l}\text { El conocimiento científico existe en el mundo que nos rodea y } \\
\text { es gracias a la capacidad que tenemos los seres humanos } \\
\text { para investigar que logramos descubrirlo en sus diferentes } \\
\text { manifestaciones. }\end{array}$ & 2 & 4 & 5 & 21 & 22 \\
\hline 13 & $\begin{array}{l}\text { Las teorías son estructuras complejas producidas por las acti- } \\
\text { vidades creadoras de la mente humana generalmente } \\
\text { después de la acumulación de una serie de datos } \\
\text { observables. }\end{array}$ & 4 & 6 & 11 & 21 & 12 \\
\hline 14 & $\begin{array}{l}\text { Los profesores deben hacer compatibles las actividades de } \\
\text { aprendizaje con las de las etapas del proceso de investigación } \\
\text { señaladas por el método científico. }\end{array}$ & 1 & 15 & 13 & 13 & 12 \\
\hline \multirow[t]{3}{*}{15} & $\begin{array}{l}\text { Los errores conceptuales deben corregirse explicando la inter- } \\
\text { pretación correcta de los mismos tantas veces como el } \\
\text { alumno lo necesite. }\end{array}$ & 6 & 13 & 11 & 14 & 10 \\
\hline & SUMATORIA & 91 & 152 & 170 & 230 & 167 \\
\hline & PORCENTAJES & $\begin{array}{c}11 . \\
2\end{array}$ & $\begin{array}{c}18 . \\
7\end{array}$ & $\begin{array}{c}21 . \\
0\end{array}$ & $\begin{array}{c}28 . \\
4\end{array}$ & 20. \\
\hline
\end{tabular}

Para interpretar los datos de la tabla, se debe partir por fijar los valores extremos, todas las afirmaciones calificadas por todos los 54 aspirantes tienen una posibilidad de ser escogidas $54 \times 15=810$ veces de tal manera que entre TD (Totalmente en Desacuerdo) y en $D$ (Desacuerdo ) fueron elegidas 30.00\% (11.23 + 18.76); Los que están entre A-D (acuerdo y Desacuerdo) $21.00 \%$ y los que están A (De Acuerdo) y T-A (Totalmente de Acuerdo) suman el $49.00 \%$.

Tabla No 2 Concepciones Epistemológicas (Frecuencias de Aceptación por ítem y promoción)

\begin{tabular}{|c|c|c|c|c|c|c|c|c|c|c|c|c|c|c|c|}
\hline $\begin{array}{c}\text { Íte } \\
\text { m }\end{array}$ & \multicolumn{4}{|c|}{$\begin{array}{c}\text { Promoción 11 } \\
\text { (frecuencia) }\end{array}$} & \multicolumn{4}{c|}{$\begin{array}{c}\text { Promoción 12 } \\
\text { (frecuencia) }\end{array}$} & \multicolumn{3}{c|}{$\begin{array}{c}\text { Promoción 13 } \\
\text { (frecuencia) }\end{array}$} \\
\hline & TD & D & $\begin{array}{c}\text { D- } \\
\text { A }\end{array}$ & A & TA & TD & D & $\begin{array}{c}\text { D- } \\
\text { A }\end{array}$ & A & TA & TD & $\begin{array}{c}\text { D } \\
\text { A }\end{array}$ & A & TA \\
\hline $\mathbf{1}$ & & 4 & 2 & 2 & 4 & & 1 & 4 & 6 & 1 & & & 5 & & 1 \\
\hline $\mathbf{2}$ & 3 & 5 & 3 & 1 & & & 8 & 1 & 1 & 2 & 1 & 3 & & 1 & 1 \\
\hline $\mathbf{3}$ & & & 1 & 7 & 4 & & 1 & 1 & 7 & 3 & & & & 1 & 5 \\
\hline $\mathbf{4}$ & 4 & 5 & 2 & & 1 & 3 & 6 & 2 & 1 & & 3 & 1 & 2 & & \\
\hline $\mathbf{5}$ & 1 & 3 & 4 & 2 & 2 & 1 & 6 & 2 & 3 & & 1 & 2 & 2 & & 1 \\
\hline $\mathbf{6}$ & 1 & 1 & 1 & 3 & 6 & 1 & 1 & 2 & 5 & 3 & & & 5 & & 1 \\
\hline $\mathbf{7}$ & 1 & 1 & 4 & 3 & 3 & & 2 & 2 & 6 & 2 & & 1 & 3 & 1 & 1 \\
\hline $\mathbf{8}$ & & 2 & 3 & 4 & 3 & & & 5 & 3 & 4 & & & 2 & 3 & 1 \\
\hline
\end{tabular}


UNIVERSIDAD PEDAGOGICA NACIONAL

\begin{tabular}{|c|c|c|c|c|c|c|c|c|c|c|c|c|c|c|c|}
\hline 9 & & 2 & 1 & 7 & 2 & & 5 & 4 & 3 & & & 2 & 2 & 2 & \\
\hline 10 & 1 & 1 & 1 & 4 & 5 & & 1 & 3 & 6 & 2 & & 1 & 1 & 1 & 3 \\
\hline 11 & 6 & 2 & 3 & 1 & & 1 & 5 & 4 & 1 & 1 & & 3 & 2 & 1 & \\
\hline 12 & & 1 & 1 & 5 & 5 & & 2 & 1 & 7 & 2 & & & & 3 & 3 \\
\hline 13 & 1 & 1 & 2 & 3 & 5 & & 2 & 2 & 6 & 2 & & & 2 & 4 & \\
\hline 14 & & 3 & 3 & 2 & 4 & 1 & 6 & 1 & 3 & 1 & & 2 & 2 & 1 & 1 \\
\hline 15 & 1 & 2 & 2 & 3 & 4 & 4 & 2 & 2 & 3 & 1 & & 4 & 1 & & 1 \\
\hline Su & 19 & 33 & 33 & 47 & 48 & 11 & 48 & 36 & 60 & 24 & 5 & 19 & 29 & 18 & 19 \\
\hline$\%$ & $\begin{array}{c}10 . \\
5\end{array}$ & $\begin{array}{c}18 . \\
3\end{array}$ & $\begin{array}{c}18 . \\
3\end{array}$ & $\begin{array}{c}26 \\
1\end{array}$ & $\begin{array}{c}26 . \\
6\end{array}$ & $\begin{array}{c}6.1 \\
1\end{array}$ & $\begin{array}{c}26 . \\
7\end{array}$ & $\begin{array}{c}20 . \\
0\end{array}$ & $\begin{array}{c}33 . \\
3\end{array}$ & $\begin{array}{c}13 . \\
3\end{array}$ & $\begin{array}{c}5.5 \\
6\end{array}$ & $\begin{array}{c}21 . \\
1\end{array}$ & $\begin{array}{c}32 . \\
2\end{array}$ & $\begin{array}{c}20 . \\
0\end{array}$ & $\begin{array}{r}21 \\
1\end{array}$ \\
\hline & \multicolumn{2}{|c|}{28.8} & $\begin{array}{c}18 \\
.3\end{array}$ & \multicolumn{2}{|c|}{52.7} & \multicolumn{2}{|c|}{32.8} & $\begin{array}{c}20 \\
.0\end{array}$ & \multicolumn{2}{|c|}{46.6} & \multicolumn{2}{|c|}{26.6} & $\begin{array}{c}32 \\
.2\end{array}$ & \multicolumn{2}{|c|}{41.1} \\
\hline
\end{tabular}

\section{Concepciones Epistemológicas (Frecuencias de Aceptación por ítem y promoción)}

\begin{tabular}{|c|c|c|c|c|c|c|c|c|c|c|c|c|c|c|c|}
\hline \multirow[t]{2}{*}{$\begin{array}{l}\text { Íte } \\
\text { m }\end{array}$} & \multicolumn{5}{|c|}{$\begin{array}{l}\text { Promoción } 14 \\
\text { (frecuencia) }\end{array}$} & \multicolumn{5}{|c|}{$\begin{array}{l}\text { Promoción } 15 \\
\text { (frecuencia) }\end{array}$} & \multicolumn{5}{|c|}{$\begin{array}{c}\text { Promoción } 16 \\
\text { (frecuencia) }\end{array}$} \\
\hline & TD & D & $\begin{array}{l}\text { D- } \\
\text { A }\end{array}$ & A & TA & TD & D & $\begin{array}{l}\text { D- } \\
\text { A }\end{array}$ & A & TA & TD & D & $\begin{array}{l}\text { D- } \\
\text { A }\end{array}$ & A & TA \\
\hline 1 & 1 & 2 & 3 & 3 & 2 & & & 2 & 1 & 2 & 2 & 1 & & 3 & 2 \\
\hline 2 & 3 & 4 & 4 & & & 2 & & & 1 & 2 & 4 & 2 & 1 & 1 & \\
\hline 3 & 1 & 1 & & 7 & 2 & & & & 2 & 3 & 1 & & & 3 & 4 \\
\hline 4 & 6 & 4 & 1 & & & 1 & 2 & 2 & & & 4 & 3 & & 1 & \\
\hline 5 & 2 & 1 & 3 & 4 & 1 & 1 & & 2 & 1 & 1 & & 2 & 1 & 4 & 1 \\
\hline 6 & 2 & 2 & 4 & & 3 & 2 & & & 1 & 2 & 1 & & 3 & 4 & \\
\hline 7 & & & 3 & 4 & 4 & & 1 & 1 & 1 & 2 & 1 & & 1 & 3 & 3 \\
\hline 8 & & & 2 & 5 & 4 & 1 & & 1 & 3 & & 1 & 1 & 2 & 3 & 1 \\
\hline 9 & & 2 & 3 & 3 & 3 & 2 & & 1 & & 2 & 3 & 1 & 2 & 2 & \\
\hline 10 & 1 & 1 & 2 & 6 & 1 & 2 & 1 & & 1 & 1 & & 1 & 2 & 4 & 1 \\
\hline 11 & 2 & 4 & 2 & 2 & 1 & 1 & & 2 & 1 & 1 & 3 & 3 & 1 & 1 & \\
\hline 12 & 1 & & 2 & 2 & 6 & 1 & & & 3 & 1 & & 1 & 1 & 1 & 5 \\
\hline 13 & 1 & 2 & 3 & 3 & 1 & & 1 & 2 & 1 & 1 & 2 & & & 4 & 2 \\
\hline 14 & & 2 & 2 & 3 & 4 & & & 2 & 1 & 2 & & 2 & 3 & 3 & \\
\hline 15 & & 4 & 1 & 4 & 2 & & & 3 & 1 & 1 & 1 & 1 & 2 & 3 & 1 \\
\hline $\mathbf{S}$ & 20 & 29 & 35 & 46 & 34 & 13 & 5 & 18 & 18 & 21 & 23 & 18 & 19 & 40 & 20 \\
\hline \multirow[t]{2}{*}{$\%$} & $\begin{array}{c}12 . \\
1\end{array}$ & $\begin{array}{c}17 . \\
6\end{array}$ & $\begin{array}{c}21 . \\
2\end{array}$ & $\begin{array}{r}27 \\
9 \\
\end{array}$ & $\begin{array}{c}20 . \\
6\end{array}$ & $\begin{array}{c}17 . \\
3\end{array}$ & 6.7 & $\begin{array}{c}24 . \\
0\end{array}$ & $\begin{array}{c}24 . \\
0\end{array}$ & $\begin{array}{c}28 . \\
0\end{array}$ & $\begin{array}{c}19 . \\
2\end{array}$ & $\begin{array}{c}15 . \\
0\end{array}$ & $\begin{array}{c}15 . \\
8\end{array}$ & $\begin{array}{c}33 . \\
3\end{array}$ & $\begin{array}{c}16 . \\
7\end{array}$ \\
\hline & \multicolumn{2}{|c|}{29.7} & 21.2 & \multicolumn{2}{|c|}{48.5} & \multicolumn{2}{|c|}{24.0} & 24.0 & \multicolumn{2}{|c|}{52.0} & \multicolumn{2}{|c|}{34.2} & 15.8 & \multicolumn{2}{|c|}{50.0} \\
\hline
\end{tabular}

Lo anterior corrobora las preferencias por las afirmaciones de corte positivista comparada por los desacuerdos con dichas afirmaciones, tan solo un $21 \%$ de estas son aceptadas a medias, es decir con dudas para rechazarlas o aceptarlas abiertamente. 


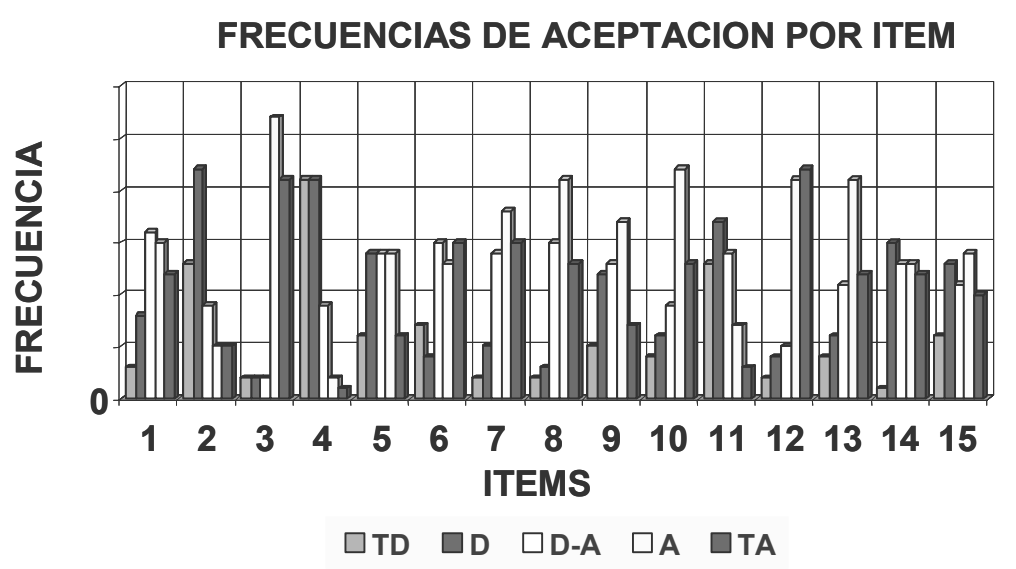

Para efectos del análisis, se pueden agrupar los datos en tres categorías, la primera conformada por los Desacuerdos y Totalmente en desacuerdo, que corresponde a la frecuencia con que son rechazadas las afirmaciones de corte empiropositivista; la segunda conformada por aquellas afirmaciones que son seleccionadas con Algo de Desacuerdo y Algo de Acuerdo que llamaré parcialmente influidas por ideas de corte empiropositivista; la tercera categoría corresponde a la frecuencia con que son aceptadas las afirmaciones de corte empiropositivistas, seleccionadas con De Acuerdo y Totalmente de Acuerdo

En la gráfica se pueden apreciar las preferencias por cada ítem según la Tabla No 2

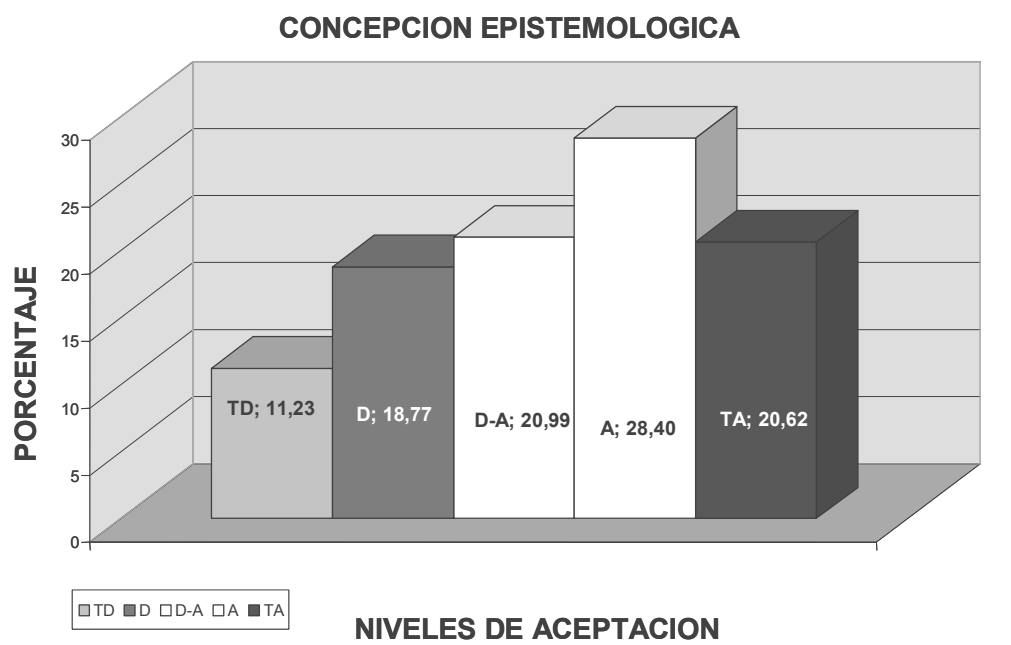

La gráfica muestra el consolidado por grado de aceptación al total de ítems del instrumento, en donde se aprecia la preponderancia de los acuerdos sobre los desacuerdos.

La tabla No 2 recoge de una manera más detallada las frecuencias del grado de aceptación por ítem y por promoción; dichos datos en términos de las tres categorías establecidas muestran que guardan correlación entre sí y que no hay variaciones significativas según promoción, al compararlas con los datos globales de la Tabla No 1, tal como se puede apreciar en la Tabla No 3.

Allí se puede observar que los valores de las desviaciones estándar son bajos, lo cual muestra que las frecuencias con que son aceptados o no cada uno de los ítems en las tres categorías por cada grupo de aspirantes de las seis promociones poseen una dispersión baja, por lo tanto los resultados se pueden evaluar como si se tratara de un solo grupo. 


\begin{tabular}{|c|c|c|c|}
\hline \multicolumn{3}{|c|}{ Tabla No 3: Niveles de aceptación por categorías y promoción } \\
\hline Promoción & $\begin{array}{c}\text { Porcentajes } \\
\text { Frecuencias } \\
\text { Desacuerdos }\end{array}$ & $\begin{array}{c}\text { Porcentajes } \\
\text { frecuencias } \\
\text { Desacuerdos y } \\
\text { Acuerdos }\end{array}$ & $\begin{array}{c}\text { Porcentajes } \\
\text { Frecuencias } \\
\text { Acuerdos }\end{array}$ \\
\hline 11 & 28.8 & 18.3 & 52.7 \\
\hline 12 & 32.8 & 20.0 & 46.6 \\
\hline 13 & 26.6 & 32.2 & 41.1 \\
\hline 14 & 29.7 & 21.2 & 48.5 \\
\hline 15 & 24.0 & 24.0 & 52.0 \\
\hline 16 & 34.2 & 15.8 & 50.0 \\
\hline Promedio & $\mathbf{3 0 . 0 0}$ & $\mathbf{2 1 . 0 0}$ & $\mathbf{4 9 . 0 0}$ \\
\hline Desv. Est. & $\mathbf{3 , 8 0}$ & $\mathbf{5 , 7 4}$ & $\mathbf{4 , 2 5}$ \\
\hline
\end{tabular}

Análisis de aceptaciones por aspirante.

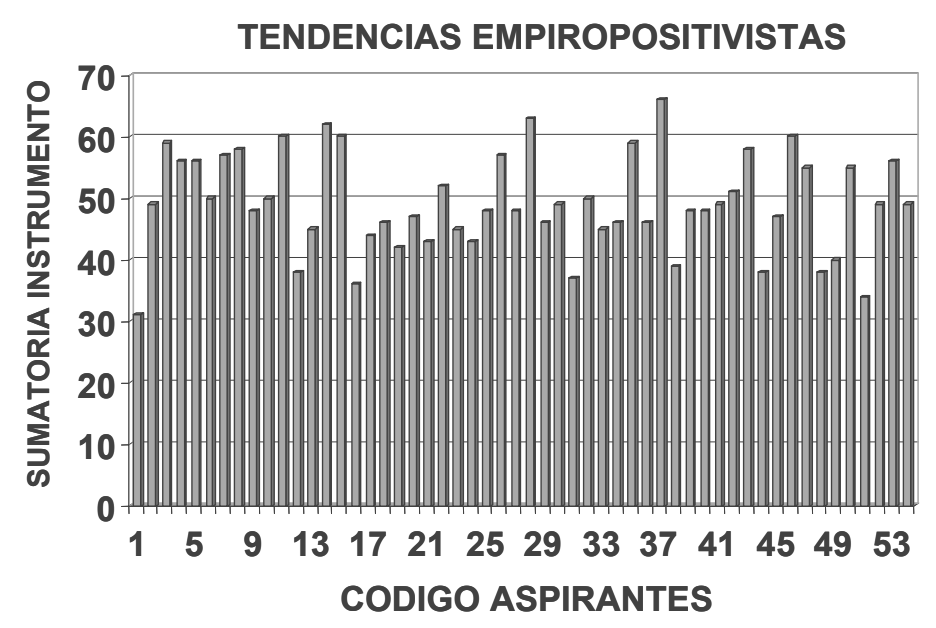

En la siguiente gráfica se puede apreciar el comportamiento de cada profesor aspirante al programa con respecto a la puntuación total (75 para los que respondan todos los ítems con totalmente de acuerdo y 15 para los que respondan todos los ítems con totalmente en desacuerdo); como entre 15 y 75 hay 60 unidades y en este caso hablamos de personas, se pueden establecer tres categorías de aspirantes: A) No influenciados por una concepción empiropositivista de la ciencia y sus procesos de producción de conocimiento,

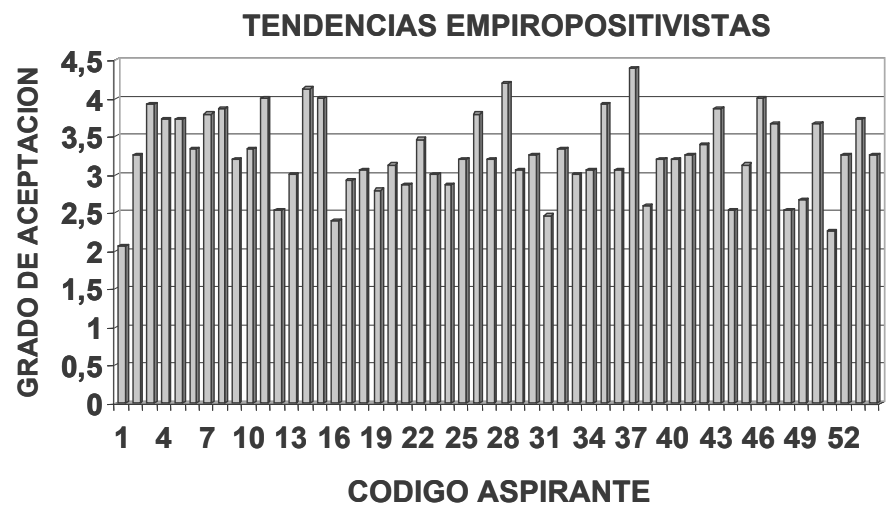
corresponde a aspirantes que hayan calificado la prueba con menos de 35 puntos; B) influenciados moderadamente cuando hayan calificado la prueba a partir de los 35 puntos y menos de 55; C): Muy influenciados por una imagen empiropositivista de la ciencia y sus procesos de producción de conocimiento aquellos cuya prueba haya sido calificada a partir de los 55 puntos. 
TABLA No 4

\begin{tabular}{|c|c|c|c|c|c|c|c|c|c|c|c|c|c|c|c|c|c|}
\hline No & I-1 & I_2 & $I-3$ & $I-4$ & I-5 & I-6 & I-7 & I-8 & I-9 & $\begin{array}{l}\text { I- } \\
10\end{array}$ & $\begin{array}{l}\text { I- } \\
11\end{array}$ & $\begin{array}{l}\text { I- } \\
12\end{array}$ & $\begin{array}{l}\text { I- } \\
13\end{array}$ & $\begin{array}{l}\text { I- } \\
14\end{array}$ & $\begin{array}{c}\text { I- } \\
15\end{array}$ & Sum & Prom \\
\hline 001 & 2 & 1 & 4 & 2 & 2 & 1 & 1 & 2 & 4 & 1 & 1 & 4 & 2 & 2 & 2 & 31 & 2,07 \\
\hline 002 & 3 & 2 & 4 & 1 & 4 & 3 & 3 & 4 & 2 & 4 & 1 & 5 & 5 & 4 & 4 & 49 & 3,27 \\
\hline 003 & 4 & 3 & 4 & 2 & 3 & 5 & 5 & 4 & 4 & 5 & 1 & 4 & 5 & 5 & 5 & 59 & 3,93 \\
\hline 004 & 5 & 4 & 4 & 3 & 3 & 5 & 4 & 4 & 4 & 3 & 3 & 4 & 4 & 3 & 3 & 56 & 3,73 \\
\hline 005 & 3 & 1 & 5 & 1 & 2 & 5 & 3 & 5 & 5 & 5 & 1 & 5 & 5 & 5 & 5 & 56 & 3,73 \\
\hline 006 & 5 & 2 & 5 & 1 & 5 & 5 & 2 & 3 & 4 & 4 & 3 & 2 & 5 & 3 & 1 & 50 & 3,33 \\
\hline 007 & 5 & 2 & 4 & 2 & 3 & 4 & 5 & 3 & 4 & 5 & 4 & 3 & 5 & 4 & 4 & 57 & 3,8 \\
\hline 008 & 2 & 3 & 5 & 5 & 2 & 5 & 4 & 5 & 4 & 5 & 1 & 4 & 3 & 5 & 5 & 58 & 3,87 \\
\hline 009 & 2 & 2 & 4 & 3 & 3 & 4 & 3 & 4 & 3 & 4 & 2 & 5 & 3 & 2 & 4 & 48 & 3,20 \\
\hline 010 & 4 & 2 & 4 & 2 & 4 & 4 & 3 & 2 & 4 & 4 & 3 & 4 & 4 & 3 & 3 & 50 & 3,33 \\
\hline 011 & 5 & 3 & 5 & 2 & 5 & 5 & 5 & 5 & 5 & 5 & 2 & 5 & 1 & 5 & 2 & 60 & 4,00 \\
\hline 012 & 2 & 1 & 3 & 1 & 1 & 2 & 4 & 3 & 2 & 2 & 1 & 5 & 4 & 2 & 5 & 38 & 2,53 \\
\hline 013 & 4 & 2 & 2 & 2 & 2 & 3 & 2 & 3 & 3 & 3 & 3 & 4 & 4 & 4 & 4 & 45 & 3,00 \\
\hline 014 & 5 & 5 & 4 & 4 & 4 & 4 & 4 & 4 & 4 & 4 & 4 & 4 & 4 & 4 & 4 & 62 & 4,13 \\
\hline 015 & 3 & 4 & 5 & 2 & 4 & 4 & 5 & 5 & 4 & 5 & 3 & 5 & 2 & 5 & 4 & 60 & 4,00 \\
\hline 016 & 2 & 2 & 4 & 3 & 2 & 1 & 2 & 3 & 2 & 2 & 3 & 4 & 3 & 2 & 1 & 36 & 2,40 \\
\hline 017 & 3 & 5 & 4 & 1 & 2 & 4 & 4 & 3 & 2 & 3 & 3 & 2 & 4 & 1 & 3 & 44 & 2,93 \\
\hline 018 & 4 & 2 & 3 & 2 & 2 & 3 & 4 & 3 & 4 & 4 & 2 & 4 & 3 & 4 & 2 & 46 & 3,07 \\
\hline 019 & 4 & 2 & 4 & 2 & 2 & 2 & 3 & 3 & 3 & 4 & 2 & 4 & 2 & 2 & 3 & 42 & 2,80 \\
\hline 020 & 4 & 2 & 5 & 1 & 3 & 5 & 3 & 5 & 2 & 5 & 2 & 3 & 4 & 2 & 1 & 47 & 3,13 \\
\hline 021 & 3 & 2 & 4 & 2 & 4 & 4 & 4 & 4 & 2 & 4 & 1 & 2 & 4 & 2 & 1 & 43 & 2,87 \\
\hline 022 & 4 & 2 & 5 & 2 & 3 & 5 & 4 & 5 & 3 & 4 & 2 & 4 & 5 & 2 & 2 & 52 & 3,47 \\
\hline 023 & 3 & 2 & 4 & 1 & 1 & 5 & 4 & 5 & 2 & 4 & 2 & 4 & 5 & 2 & 1 & 45 & 3,00 \\
\hline 024 & 3 & 2 & 4 & 1 & 1 & 3 & 3 & 4 & 2 & 2 & 4 & 4 & 4 & 4 & 2 & 43 & 2,87 \\
\hline 025 & 3 & 2 & 5 & 1 & 5 & 3 & 3 & 4 & 2 & 5 & 2 & 4 & 4 & 3 & 2 & 48 & 3,20 \\
\hline 026 & 4 & 3 & 4 & 3 & 2 & 4 & 5 & 4 & 3 & 3 & 5 & 5 & 4 & 3 & 5 & 57 & 3,80 \\
\hline 027 & 3 & 4 & 5 & 3 & 3 & 3 & 4 & 4 & 3 & 3 & 2 & 4 & 3 & 2 & 2 & 48 & 3,20 \\
\hline 028 & 3 & 5 & 5 & 3 & 2 & 5 & 5 & 5 & 4 & 5 & 3 & 5 & 3 & 5 & 5 & 63 & 4,20 \\
\hline 029 & 5 & 2 & 5 & 1 & 2 & 3 & 2 & 3 & 3 & 4 & 2 & 5 & 4 & 3 & 2 & 46 & 3,06 \\
\hline 039 & 3 & 1 & 5 & 2 & 3 & 3 & 3 & 3 & 4 & 5 & 3 & 5 & 4 & 2 & 3 & 49 & 3,27 \\
\hline 031 & 3 & 1 & 4 & 1 & 2 & 1 & 3 & 4 & 2 & 2 & 1 & 3 & 2 & 3 & 5 & 37 & 2,47 \\
\hline 032 & 4 & 3 & 1 & 2 & 4 & 1 & 5 & 4 & 4 & 4 & 1 & 5 & 5 & 5 & 2 & 50 & 3,33 \\
\hline 033 & 3 & 1 & 4 & 1 & 1 & 5 & 3 & 3 & 3 & 4 & 4 & 4 & 3 & 4 & 2 & 45 & 3,00 \\
\hline 034 & 3 & 2 & 4 & 1 & 4 & 3 & 4 & 4 & 4 & 3 & 4 & 1 & 4 & 3 & 2 & 46 & 3,07 \\
\hline 035 & 5 & 3 & 4 & 2 & 4 & 3 & 4 & 5 & 3 & 4 & 3 & 5 & 4 & 5 & 5 & 59 & 3,93 \\
\hline 036 & 4 & 3 & 4 & 1 & 3 & 2 & 3 & 4 & 3 & 3 & 2 & 4 & 4 & 2 & 4 & 46 & 3,07 \\
\hline 037 & 5 & 3 & 5 & 1 & 5 & 5 & 5 & 5 & 5 & 5 & 5 & 5 & 3 & 4 & 5 & 66 & 4,40 \\
\hline 038 & 1 & 1 & 4 & 3 & 4 & 2 & 4 & 3 & 5 & 1 & 2 & 5 & 0 & 0 & 4 & 39 & 2,60 \\
\hline 039 & 2 & 2 & 5 & 1 & 1 & 5 & 5 & 5 & 5 & 4 & 2 & 5 & 2 & 2 & 2 & 48 & 3,20 \\
\hline 040 & 2 & 2 & 2 & 2 & 3 & 3 & 5 & 5 & 4 & 4 & 2 & 5 & 1 & 5 & 3 & 48 & 3,20 \\
\hline 041 & 4 & 2 & 4 & 2 & 3 & 3 & 4 & 4 & 2 & 4 & 3 & 3 & 3 & 4 & 4 & 49 & 3,27 \\
\hline
\end{tabular}




\begin{tabular}{|c|c|c|c|c|c|c|c|c|c|c|c|c|c|c|c|c|c|}
\hline $\mathbf{0 4 2}$ & 5 & 4 & 5 & 2 & 3 & 4 & 3 & 4 & 1 & 2 & 4 & 4 & 4 & 3 & 3 & 51 & 3,40 \\
\hline $\mathbf{0 4 3}$ & 5 & 5 & 5 & 3 & 3 & 1 & 4 & 4 & 5 & 5 & 3 & 4 & 3 & 5 & 3 & 58 & 3,87 \\
\hline $\mathbf{0 4 4}$ & 3 & 1 & 4 & 1 & 4 & 1 & 2 & 1 & 3 & 1 & 1 & 5 & 3 & 3 & 5 & 38 & 2,53 \\
\hline $\mathbf{0 4 5}$ & 3 & 1 & 5 & 3 & 1 & 5 & 5 & 3 & 1 & 1 & 5 & 1 & 5 & 5 & 3 & 47 & 3,13 \\
\hline $\mathbf{0 4 6}$ & 4 & 5 & 4 & 2 & 5 & 5 & 5 & 4 & 5 & 4 & 3 & 4 & 2 & 4 & 4 & 60 & 4,00 \\
\hline $\mathbf{0 4 7}$ & 5 & 1 & 4 & 2 & 4 & 4 & 5 & 5 & 2 & 4 & 4 & 5 & 4 & 2 & 4 & 55 & 3,67 \\
\hline $\mathbf{0 4 8}$ & 4 & 1 & 1 & 1 & 5 & 1 & 4 & 1 & 1 & 5 & 2 & 5 & 1 & 3 & 3 & 38 & 2,53 \\
\hline $\mathbf{0 4 9}$ & 1 & 2 & 5 & 1 & 3 & 3 & 1 & 3 & 4 & 3 & 1 & 5 & 4 & 3 & 1 & 40 & 2,67 \\
\hline $\mathbf{0 5 0}$ & 4 & 2 & 5 & 2 & 4 & 4 & 5 & 4 & 3 & 4 & 2 & 4 & 4 & 4 & 4 & 55 & 3,67 \\
\hline $\mathbf{0 5 1}$ & 1 & 1 & 4 & 1 & 2 & 3 & 3 & 3 & 4 & 2 & 3 & 2 & 1 & 2 & 2 & 34 & 2,27 \\
\hline $\mathbf{0 5 2}$ & 2 & 1 & 4 & 4 & 4 & 4 & 4 & 2 & 1 & 4 & 1 & 5 & 5 & 4 & 4 & 49 & 3,27 \\
\hline $\mathbf{0 5 3}$ & 5 & 4 & 5 & 1 & 4 & 4 & 5 & 4 & 1 & 4 & 1 & 5 & 4 & 4 & 5 & 56 & 3,73 \\
\hline $\mathbf{0}$ & 4 & 3 & 5 & 2 & 2 & 3 & 4 & 4 & 3 & 3 & 2 & 3 & 5 & 3 & 3 & 49 & 3,27 \\
$\mathbf{5 4}$ & & & & & & & & & & & & & & & & & \\
\hline
\end{tabular}

A continuación se presenta una breve discusión de los datos presentados en la Tabla No 4 consistente en observar como varían los resultados en tres análisis después de modificar en algunas unidades los rangos elegidos para cada una de las tres categorías, sobre concepciones de ciencia y los procesos de producción de conocimiento, previamente establecidas.

\begin{tabular}{|c|c|c|c|c|c|c|}
\hline \multicolumn{7}{|c|}{ TABLA No 5 } \\
\hline \multirow{2}{*}{ Categoría } & \multicolumn{2}{|c|}{ Análisis 1 } & \multicolumn{2}{c|}{ Análisis 2 } & \multicolumn{2}{c|}{ Análisis 3 } \\
\cline { 2 - 7 } & Rango 1 & $\begin{array}{c}\text { Resultado } \\
1\end{array}$ & Rango 2 & $\begin{array}{c}\text { Resultado } \\
2\end{array}$ & Rango 3 & $\begin{array}{c}\text { Resultado } \\
3\end{array}$ \\
\hline A & $15-34$ & 2 & $15-38$ & 7 & $15-39$ & 8 \\
\hline B & $35-54$ & 36 & $39-52$ & 30 & $40-54$ & 29 \\
\hline C & $55-75$ & 16 & $53-75$ & 17 & $55-75$ & 17 \\
\hline
\end{tabular}

De acuerdo con la Tabla No 5, basado en el Análisis 1, la categoría A correspondiente a los profesores que definitivamente se puede decir no muestran influencias empiropositivistas de la ciencia y sus procesos de producción de conocimiento son 2; categoría $\mathrm{B}$, o sea los que manifiestan alguna influencia son la mayoría, 36 y los de la categoría $C$, los que están altamente influenciados por estas corrientes filosófico epistemológicas son 16.

Al ampliar el rango de la categoría A análisis 2 y 3 se observa que aumenta en 5 o 6 profesores respectivamente, llegando al $13 \%$, afectando la categoría $B$ en ese número de profesores, la cual continúa siendo significativamente la más alta. 55,5\%

Al modificar los rangos para la categoría $\mathrm{C}$, se observa que prácticamente permanece alrededor de 16 o 17 profesores que corresponden al $31,5 \%$

\section{CONCLUSIONES}


Los aspirantes muestran marcada aceptación a las siguientes afirmaciones, las cuales superaron el $50 \%$ de las preferencias individuales y el 65 las eligió entre De Acuerdo y Totalmente de Acuerdo:

- La observación es el primer paso del método científico y está relacionada con la forma como percibimos, con los órganos de los sentidos, el mundo que nos rodea, esto nos permite admitir la seguridad en nuestras apreciaciones y la confiabilidad en los resultados. (27/54)

- La actividad investigativa gira alrededor de un hecho o fenómeno que ocurre en la realidad y que amerita una explicación. (48/54)

- Los conceptos y las teorías son producidos por actos creativos que surgen directamente de la observación y experimentación rigurosa de la realidad.(28/54)

- Las explicaciones científicas pueden ser aceptadas como verdaderas solamente si ellas pueden ser comprobadas por observación y/o la experimentación. (33/54)

- Las teorías son el producto de la experimentación y el experimento es necesario para darle sentido a las observaciones de eventos naturales. (34/54)

- Un principio que debe reconocer el profesor de ciencias es que la observación y la experimentación son los procesos más confiables y seguros a partir del cual se elaboran las teorías. (35/54)

- El conocimiento científico existe en el mundo que nos rodea y es gracias a la capacidad que tenemos los seres humanos para investigar que logramos descubrirlo en sus diferentes manifestaciones. (43/54)

- Las teorías son estructuras complejas producidas por las actividades creadoras de la mente humana generalmente después de la acumulación de una serie de datos observables. $(33 / 54)$

De acuerdo con la hipótesis planteada, se puede afirmar que en relación con las concepciones que el profesor ha construido sobre la Ciencia y los procesos de producción de conocimiento científico (la Investigación), se encontró que el $31.5 \%$ de los aspirantes al programa se encuentran altamente influenciados por concepciones ingenuas e intuitivas así como inductivista-experimental. El 55.5\% manifiesta estar influenciado por el inductivismo experimental y tan solo el $13 \%$ no está claramente influenciado por estas corrientes epistemológicas.

\section{BIBLIOGRAFIA}

1. Erazo Parga M., "El Pensamiento del Profesor" Influencias empiropositivistas en las concepciones de Investigación, Ciencia, Epistemología, Enseñanza, Aprendizaje y Modelo Didáctico en profesores de Ciencias del nivel básico. Universidad Pedagógica Nacional. 1999.

2. Erazo Parga M., "Hacia una Enseñanza de la Ciencias por Investigación" Páginas 37-44. Revista Educación y Cultura de FECODE. Nº 38 Agosto de 1995.

3. Erazo Parga M., Alternativas Epistemológicas y Didácticas en relación con la Enseñanza y el Aprendizaje de las Ciencias. Revista DIOGENES Vol. 4 № 2 Universidad De La Salle. Pág. 245-259.

4. Erazo Parga M., Consideraciones críticas para la aproximación hacia un modelo de enseñanza - aprendizaje de las ciencias por Investigación. Investigación en la Escuela. N12, 1990. Universidad de Sevilla. España. 
5. Escudero, E.T. Y Lacasta. Z.E., 1984. Las Actitudes Científicas de los Maestros en Relación con sus Conocimientos. Enseñanza de las Ciencias. 2 (3). pp 175-180.

6. Fernández, I; Gil, D; Carrascosa,J; Cachapuz A. Visiones Deformadas de la ciencia transmitidas por la enseñanza. Enseñanza de las Ciencias 2002, 20 (3) pp 477-488.

7. García Marcelo. C El pensamiento del profesor. CEAC Barcelona 1987.

8. Goodwin A; Orlik Y. 2002. Investigación sobre la comprensión de los conceptos de evaporación y ebullición que poseen los profesores de ciencias. Educación en Ciencias Vol $1 \mathrm{~N}^{\circ} 2$.

9. Martínez Aznar y Otros. ¿Qué pensamiento profesional y curricular tienen los futuros profesores de ciencias de secundaria?. Enseñanza de las Ciencias, 2001, 19 (1).

10. Mellado, V 1999. La investigación sobre la formación del profesorado de Ciencias Experimentales. En C. Martinez y S García: La didáctica de las ciencias. Tendencias actuales. U de Coruña. Pp 45-76.

11. Porlán Rafael. El Pensamiento Científico y Pedagógico de Maestros en Formación. Constructivismo y Enseñanza de las ciencias DIADA 1988 Pág. 193.

12. Pozo J. I El adolescente como científico. Cuadernos de Pedagogía No 151 Pág. 74 a 78. 1987. 